\title{
Atomic and Electronic Structure of $\mathrm{SrTiO}_{3} / \mathrm{GaAs}$ Hetero-Interfaces
}

\author{
Q. Qiao, R.F. Klie, S. Ogut \\ Department of Physics, University of Illinois at Chicago, Chicago, IL 60607
}

Metal-oxide semiconductor interfaces have received much attention in recent years due to their potential applications in metal-oxide-semiconductor field-effect transistors. In particular, crystalline metal-oxide/semiconductor systems have emerged as a possible alternative to the current silicabased technologies. As a result, extensive efforts have been made to find thermodynamically and electrically stable insulator/semiconductor interfaces and a number of single-crystal metal-oxide films have now been grown. In this study, we aim at developing a fundamental model of singlecrystal $\mathrm{SrTiO}_{3} / \mathrm{GaAs}$ interfaces by examining the atomic and electronic structures of epitaxial $\mathrm{SrTiO}_{3}[100]$ thin films on $\mathrm{GaAs}[001]$ using atomic-resolution Z-contrast imaging and electron energy-loss spectroscopy in combination with first principles calculations.

Initially, two different kinds of ultra-thin $\mathrm{SrTiO}_{3}$ films were deposited on As-terminated GaAs (100), one with a Ti pre-layer and the other with the $\mathrm{SrTiO}_{3}$ in direct contact with the GaAs support [1]. Figure 1 shows high-resolution Z-contrast images using an aberration-corrected scanning transmission electron microscope of the two different $\mathrm{SrTiO}_{3}$ films. The images suggest that the oxide film starts with a SrO layer at the As terminated GaAs interface. As shown in Figure 2, the $\mathrm{SrTiO}_{3} / \mathrm{GaAs}(001)$ interface is then modeled from first principles starting with the simplest possible interface consistent with these Z-contrast images, but the interfaces using stoichiometric $\mathrm{SrTiO}_{3}$ unit cells provide conflicting results compared to the experimental observations. In Figure 3 we demonstrate that by taking into consideration the $\mathrm{SrTiO}_{3} /$ vacuum interface, the calculations of two non-stoichiometric oxide films find the As-SrO to be preferable to the GaAs/oxide interface, in agreement with our Z-contrast imaging study.

However, the electronic structure of these simple interfaces, in which we used a (1x1) GaAs surface unit cell does not match the measured electrical properties of the thin film. More specifically, our calculations show that interfacial states are introduced in the band-gap, while photoemission data on the $\mathrm{SrTiO}_{3} / \mathrm{GaAs}(001)$ interface show a clean band-gap suggesting dielectric nature [2], and the measured valence-band-offset (VBO) is near $2.5 \mathrm{eV}$. In contrast, our calculated VBO with a (1x1) unit cell find a $\mathrm{VBO}$ of $\sim 1 \mathrm{eV}$. By making use of one feature of the Z-contrast images, we try to find how different $\operatorname{GaAs}(001)$ surface reconstructions are modified in the presence of $\mathrm{SrTiO}_{3}$. Since it is impossible to tell from the images in Figure 1 whether some of the sites assigned to $\mathrm{O}$ atoms are indeed fully occupied by them, we can assume there to be oxygen vacancies at the interface. First principles total energy calculations are used to find the low-energy semiconducting interface. Band offsets of any model proposed by the modeling will be compared to the experimental results to verify the validity of or discard the proposed structures.

In this presentation, we will utilize atomic-resolution Z-contrast imaging and EELS in combination with first-principles calculations to show that the As-SrO interfaces are energetically favorable and surface reconstructions might cause a low-energy semiconducting interface. In addition, we will explore the role of $\mathrm{O}$ vacancies and Ti diffusion in stabilizing the semiconducting interface. [3] 
References

[1] R.F. Klie et al., Appl. Phys. Lett. 87 (14) (2005) 143106.

[2] Y. Liang et al., Appl. Phys. Lett. 86 (8) (2005) 082905.

[3] This research was supported by the National Science Foundation under Grant No. DMR0604964, and also supported by the NSF CAREER Award DMR-0846748.

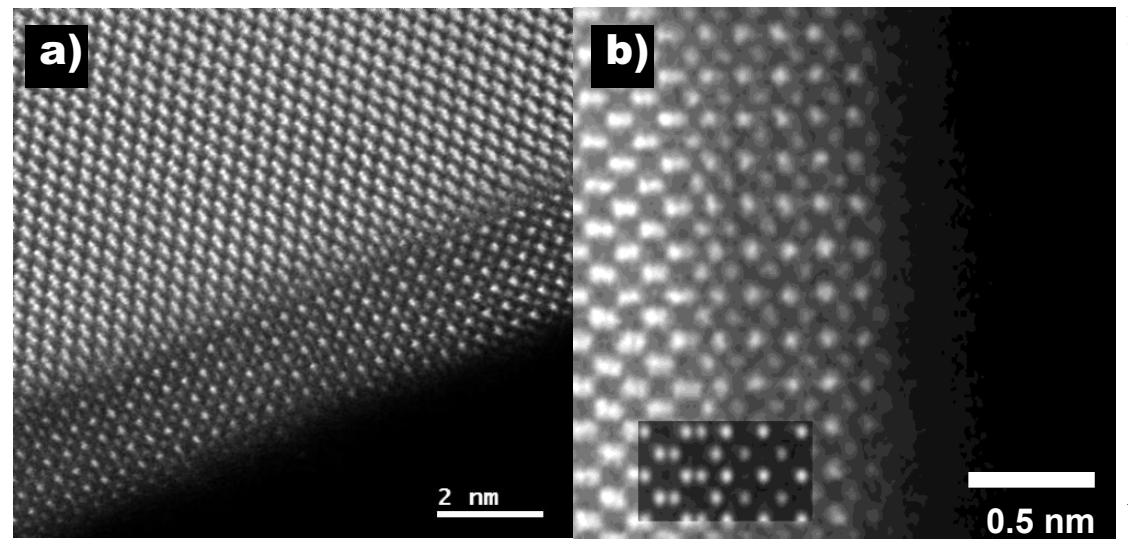

FIG. 1. Z-contrast images of the $\mathrm{SrTiO}_{3}$ film on GaAs for two different growth conditions. The $\mathrm{SrTiO}_{3}$ is shown in the [001] orientation while the GaAs is seen along the [110] in both samples. a) $\mathrm{SrTiO}_{3}$ film deposited on GaAs after depositing a Ti pre-layer; b) same growth conditions for $\mathrm{SrTiO}_{3}$ except that no Ti-prelayer was deposited [1].
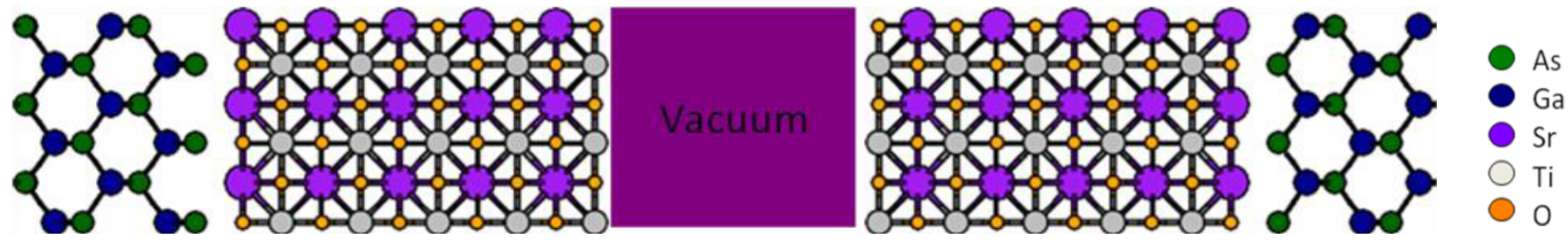

FIG. 2. First Principles modeling of $\mathrm{SrTiO}_{3} / \mathrm{GaAs}$ interfaces: Calculation is based on PAW method within DFT 3x3x1 K-points, 300 eV cutoff. Slab geometry: Symmetric double slabs with 6/7 atomic layers of Ga/As, 1-5 Stoichiometric $\mathrm{SrTiO}_{3}$ units, 8/10 $\AA$ Vacuum and 1x1 Surface cell (3x3 shown)

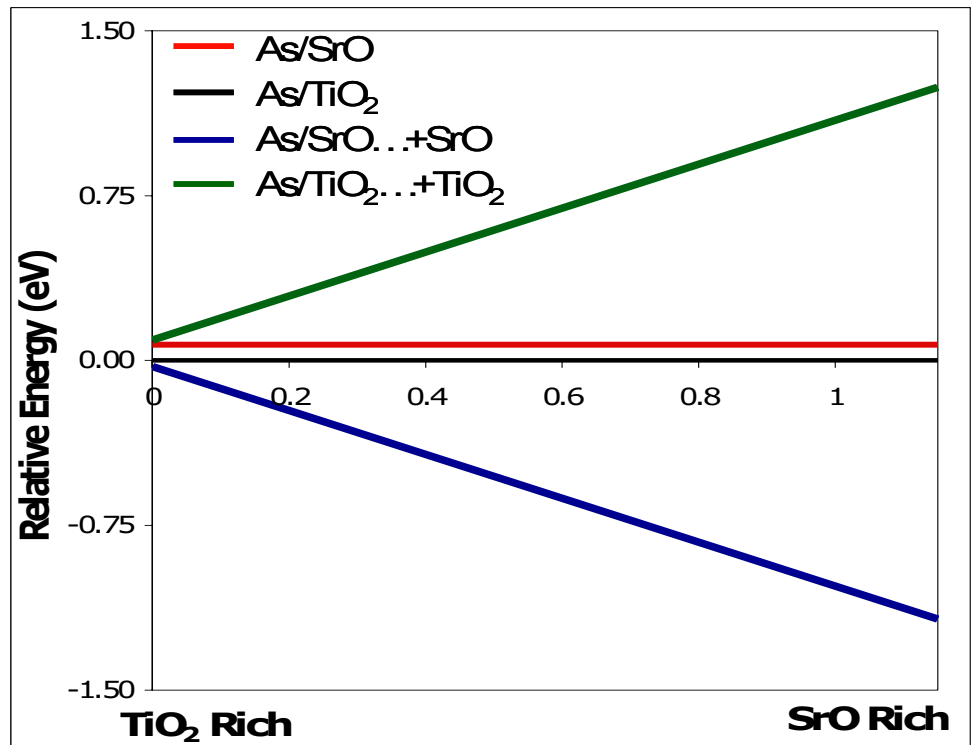

FIG. 3. Energetics of the $\mathrm{SrTiO}_{3} / \mathrm{GaAs}$ interface using the PAW method within DFT for different interfacial configurations. These calculations clearly show that the $\mathrm{SrTiO}_{3}$ film starting with the $\mathrm{SrO}$ layer is lower in energy over the entire range of the chemical potential than the $\mathrm{SrTiO}_{3}$ starting with the $\mathrm{TiO}_{2}$ layer. Nonstoichiometric $\mathrm{SrTiO}_{3}$ films were used since the $\mathrm{SrTiO}_{3} /$ vacuum interface contributes significantly to the overall formation energy. 